\title{
TBI Technique Improvements for Anesthetized Pediatric Patients Based on Near-Miss Incident Reporting
}

\author{
Ning Cao ${ }^{1 *}$, Eric C. Ford ${ }^{1}$, Matthew J. Nyflot ${ }^{1}$, Ralph P. Ermoian${ }^{1}$, Nicole W. Pelly ${ }^{2}$, Lori Young ${ }^{1}$, \\ Kristi Hendrickson 1 \\ ${ }^{1}$ Department of Radiation Oncology, University of Washington, Seattle, WA, USA \\ ${ }^{2}$ Department of Anesthesiology \& Pain Medicine, University of Washington, Seattle, WA, USA \\ Email:ningcao@uw.edu
}

How to cite this paper: Cao, N., Ford, E.C., Nyflot, M.J., Ermoian, R.P., Pelly, N.W., Young, L. and Hendrickson, K. (2016) TBI Technique Improvements for Anesthetized Pediatric Patients Based on Near-Miss Incident Reporting. International Journal ot Medical Physics, Clinical Engineering and Radiation Oncology, 5, 270-280.

http://dx.doi.org/10.4236/ijmpcero.2016.54027

Received: August 13, 2016

Accepted: November 7, 2016

Published: November 10, 2016

Copyright $\odot 2016$ by authors and Scientific Research Publishing Inc. This work is licensed under the Creative Commons Attribution International License (CC BY 4.0).

http://creativecommons.org/licenses/by/4.0/

(c) (i) Open Access

\begin{abstract}
Purpose: To share our clinical experience of an optimized and comprehensive pediatric TBI technique. Methods and Materials: Through the use of incident learning, safety-critical areas were identified in our procedure for total body irradiation (TBI) for pediatric patients under anesthesia for bone-marrow transplant. The previous procedure lacked flexibility to accommodate various requests from the anesthesia team due to the wide range of patient sizes. To address this issue and to improve the process overall, we updated our procedure for TBI simulation, dosimetry planning, patient setup during treatment, and in vivo dosimetry. A simulation form was redesigned with additional detailed instructions and documentation requirements. The dose calculation procedure was reformulated to remove dependence on setup variations. Tissue compensation determination and therefore dose uniformity were improved by introducing rigorous calculation methods. Calculations were performed on 28 previously-treated patients to compare the dose uniformity using the new versus previous methods. Results: The new procedures improve interdepartmental communication, simplify the workflow, decrease the risk of treating patients in a setup that differs from that used during the simulation, and reduce dose heterogeneity. The new compensator design significantly improved patient dose uniformity: $0.8 \% \pm 0.4 \%$ (new method) vs. $4.2 \% \pm 2.3 \%$ (previous method) $(\mathrm{p}<0.01$ ). Conclusion: A near-miss incident reporting system was used to improve the safety and quality of pediatric TBI procedures under anesthesia.
\end{abstract}

\section{Keywords}

Pediatric Total Body Irradiation Simulation, Dosimetry, Treatment Setup,

Continuous Safety Improvement (CSI) 


\section{Introduction}

Total body irradiation (TBI) is a form of radiotherapy technique primarily used as a conditioning regimen for hematopoietic stem cell transplant (HSCT) [1]. For certain HSCTs in pediatric patients, especially acute lymphoblastic leukemia (ALL), TBI is proven to be an effective preparatory regimen [2] [3]. TBI is most commonly used in transplantation conditioning for malignant conditions such as leukemia and lymphoma but is also used in transplant conditioning for non-malignant disease, such as common variable immunodeficiency and aplastic anemia [1].

TBI together with chemotherapeutic agents serves to ablate the patients' hematopoietic stem cells (myeloablative TBI) or to suppress the recipient's immune system prior to HSCT (non-myeloablative TBI). In addition, high doses of TBI can eradicate residual cancer cells in the recipient [1]. Myeloablative TBI is usually given in multiple fractions twice a day during a period of 3 - 5 days, with a total radiation dose of 1200 1550 cGy [4]. In our clinic, the standard pediatric TBI regimen is 1320 cGy (165 $\mathrm{cGy} /$ fraction $\times 8$ fractions) delivered in 4 days. In some circumstances, "mini” TBI (i.e., a single fraction of $200 \mathrm{cGy}$ ) is used to suppress the transplant recipient's immune system rather than to ablate the hematopoietic stem cells.

Techniques for TBI have been in existence for decades. There are a few good guidelines for TBI practice in general, such as the authoritative report, American Association of Physicists in Medicine (AAPM) Task Group 29, dates to 1986 [5] and Chapter 15 of Perez and Brady's Principles and Practice of Radiation Oncology (edition 6) [6]. However, there is relatively little information in the medical physics literature about best practices for pediatric TBI cases under anesthesia and such cases are uncommon except in a few referral centers. On average, there is one pediatric TBI under anesthesia every month and a half in our clinic. These are complex cases requiring administration of anesthesia and close collaboration of numerous professional groups. Motivation for improving our pediatric TBI procedures and processes came from a series of near-miss incidents observed through the departmental incident learning system. As a result, an in-depth review of pediatric TBI procedures was undertaken to improve the safety and quality of patient treatment using an investigation of past events and input from involved staff. This report describes the pediatric TBI treatment technique and highlights changes made in response to observed near-miss incidents. In addition, a new tissue compensation procedure was developed to improve dose uniformity as measured with in vivo dosimetry. Furthermore, new measurements of the off-axis ratios (OAR) of the treatment field suggest that the patient should be placed within an appropriate limited area indicated by the light field to avoid placing the patient within the penumbra region.

\section{Materials and Methods}

The study has been reviewed and approved by the University of Washington Institutional Review Board (IRB). 


\subsection{Departmental Incident Learning and the Need for Process Improvement}

A departmental near-miss incident reporting system was launched in February 2012. All incidents are reviewed weekly by a multi-disciplinary team of physicians, medical physicists, medical dosimetrists, radiation therapists, nurses, and administrative staff. We reviewed 1507 reports from 2/1/2012 to 10/31/2013 for reports categorized as "pediatrics" or "TBI" or containing the text string "ped*tbi", generating a total of 102 reports. Of these, five reports were chosen for root cause analysis. For example, one nearmiss incident report indicated that a 5\% potential error in dose calculation could have resulted from a non-standard set up, where the monitor unit (MU) calculation method did not automatically account for the variation in setup, requiring manual re-computation.

Motivated by these near-miss incidents, a systematic review of the previous pediatric TBI procedures was conducted, and the following issues were identified: insufficient communication describing variations in setup at simulation versus treatment related to anesthesia requirements, confusion about measurement units, unclear criteria for employing various setup options to accommodate patients ranging in age from infancy to 7 years, and variations in compensator design methods.

\subsection{Pediatric TBI Procedure Overview}

In our clinic, pediatric TBI patients requiring anesthesia are treated with an anteriorposterior/posterior-anterior (AP/PA) technique using a modern linear accelerator (linac) with gantry at $0^{\circ}$. The whole pediatric TBI treatment process includes four major components: simulation, dosimetry planning, treatment, and in vivo dosimetry. Anesthesia is provided by pediatric anesthesiologists from Seattle Children's Hospital.

\subsection{Simulation}

Although all patients treated with this technique are treated under anesthesia, anesthesia often is not used in simulation for "mini" TBI treatments because the simulation procedure is much faster and allows for technicians to gently guide the patient. The radiation oncologist, anesthesiologist, nurse, and simulation therapists decide together whether anesthesia is necessary to obtain reproducible and accurate measurements for dosimetry planning. With few exceptions, anesthetized patients receive a total intravenous anesthetic with oxygen by nasal cannula (as opposed to by laryngeal mask airway or endotracheal tube as airway support).

Simulation is performed in a CT simulator if Cerrobend lung blocks are prescribed typically only in myeloablative TBI. CT scans are then taken in the supine and prone positions to facilitate the drawing of lung blocks, which are used to limit the total dose to the lung. If lung blocks are not prescribed, simulation measurements are still obtained in the simulator suite because it is designed to allow for anesthesia equipment.

As shown in Figure 1, the patient lies on a $7-\mathrm{cm}$ pad. Patient length as well as the anterior-to-posterior separation of different parts of the body (head, neck, umbilicus, 


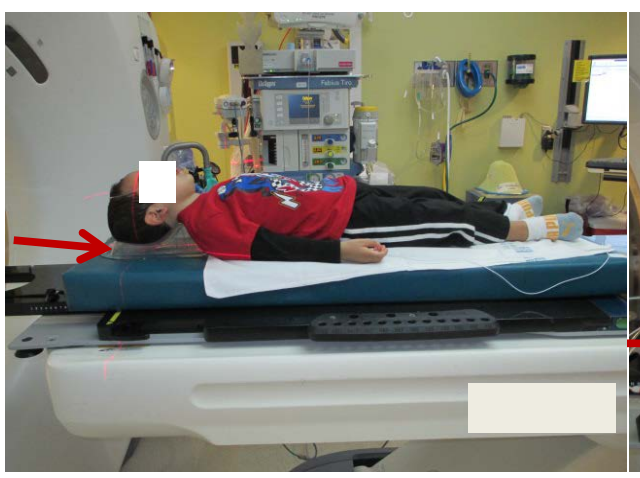

(a)

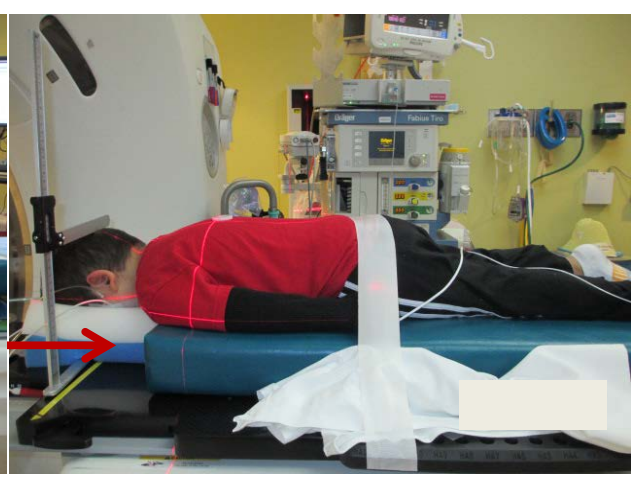

(b)

Figure 1. The photos show the simulation set-up for supine (a) and prone (b) treatment positions. Arrows point to the standard headrests used in AP and PA positions.

thigh, knee and ankle) are measured under the same setup conditions that will be used during the treatment. Whether the patient is treated within one field (no-gap, collimator at $45^{\circ}$ ) or two fields (gapped, collimator at $0^{\circ}$ ) is determined by patient length and the largest physical field size possible under treatment conditions.

\subsection{Dosimetry Planning}

Pediatric TBI is performed on a linac (Elekta Synergy; Elekta Inc., Crawley, UK) with 6-MV photon beams. The dosimetric planning for pediatric TBI is calibrated with the source-skin distance (SSD) setup. Output factor (cGy/MU) and percent depth dose (PDD) data were measured with a 0.6-cc Farmer ionization chamber in solid water under treatment conditions (field size $40 \times 40$ at isoplane and at SSD of $190 \mathrm{~cm}$ ). The dose prescription point is the midline of the thickest part of the body, which is usually the head for pediatric patients. The MUs are calculated using Equation (1). Dose related to image is not taken account into the total prescribed dose.

$$
\mathrm{MU}=\frac{\text { Prescription Dose }(\mathrm{cGy})}{\text { Output factor }\left(\frac{\mathrm{cGy}}{\mathrm{MU}}\right) \times \mathrm{PDD} \times \mathrm{SSD} \text { factor }}
$$

If the patient length (straight or frogged legs) is within $80 \mathrm{~cm}$, the patient will be treated using a one-field (no-gap) technique. Patients longer than approximately $80 \mathrm{~cm}$ will not fit within a single field and will be treated with two fields (gapped technique). For gapped fields, there will be two prescription points: the head for the superior field and the upper thigh for the inferior field since upper thigh is the thickness part in the inferior field. The gap distance is calculated based on Equation (2), where FS is the field size $(40 \mathrm{~cm}), d_{\text {thigh }}$ is the thickness of the thigh, and SAD is the distance from source to machine isocenter, which is $100 \mathrm{~cm}$.

$$
\mathrm{Gap}(\mathrm{cm})=2 \times \frac{\left(\frac{\mathrm{FS}}{2}\right) \times\left(\frac{d_{\text {thigh }}}{2}\right)}{\mathrm{SAD}}
$$


The gap is planned in the upper thigh area and its location is moved about $1 \mathrm{~cm}$ for each treatment fraction to minimize the possible hot or cold spots caused by the junction fields.

Layers of acrylic $\left(1.18 \mathrm{~g} / \mathrm{cm}^{3}\right)$ compensator are used to compensate for the variation in patient thickness, improving dose uniformity. The MU and compensator calculation is always prepared for the gapped and non-gapped techniques when patient length (straight legs) is over $80 \mathrm{~cm}$. The final setup technique is decided in the treatment room.

For myeloablative (fractionated) TBI, lung blocks made of 2 half value layer (HVL) of cerrobend are used for the first half treatment course. No lung blocks are used for non-myeloablative TBI treatment. Based on the treatment protocols and our standard of practice, shielding for other sites, such as lens, gonad and thymus, is not used. Shielding for kidney will be used only when the patients have previous radiation treatment or very poor renal function.

\subsection{Treatment}

In order to provide fields as large as possible, pediatric TBI patients are treated lying on a wooden stand that is $15 \mathrm{~cm}$ above the floor. A beam spoiler rests on the top of the stand between the patient and gantry (Figure 2). The treatment position duplicates that at simulation, including the arrangement of the support accessories (headrest, pad, etc.). Acrylic compensators and the lung blocks are placed on top of the beam spoiler. Films to verify lung block placement are acquired by placing cassettes under the table.

\subsection{In Vivo Dosimetry}

Optically-stimulated luminescence (OSL) devices are used to assess patient dose due to

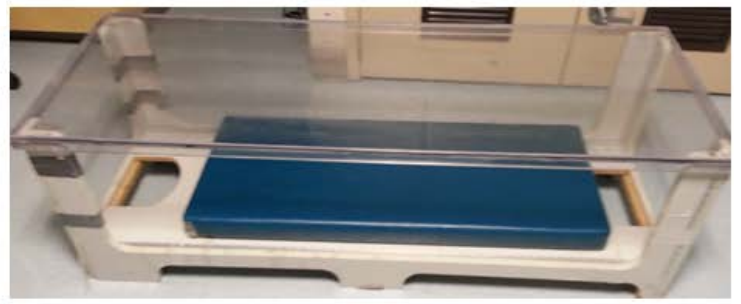

(a)

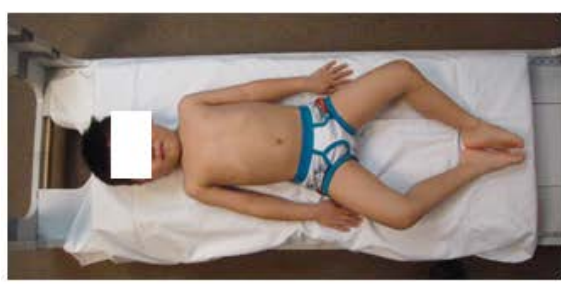

(b)

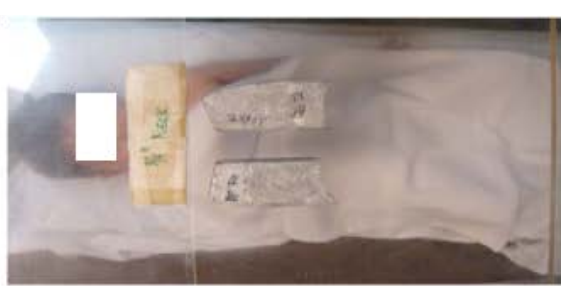

(c)

Figure 2. The photos show (a) the wooden stand with the pad and the spoiler; (b) the treatment setup in the wooden stand with frogged legs; and (c) the placement of compensators and Cerrobend lung blocks in the spoiler above the patient. 
its characteristics of energy, temperature, and angular independence [7]. OSLs with $1-\mathrm{cm}$ bolus as buildup are placed on the forehead, lung (with or without lung blocks), umbilicus, thigh, knee, and ankle. Due to the concerns with compromising patient airway under anesthesia, neck dose is not assessed. For myeloablative (fractionated) TBI, only one set of OSLs are needed to measure the daily dose, usually during the first fraction of the treatment. For non-myeloablative TBI treatment, two sets of OSLs are used, placed simultaneously on the anterior and posterior sides of the body. During non-myeloablative TBI treatments, OSLs will be read immediately after the AP beam to determine dose uniformity. Action will be taken if results are greater than $\pm 10 \%$ of the expected dose.

\section{Results}

\subsection{The Implementation of the Revised Simulation Form}

In the previous procedure, the patient setup was not well standardized and was inadequately communicated. To address this need, we redesigned a simulation form with detailed instructions and documentation requirements. The simulation staff are required to fill in all of the information in the simulation form (Figure 3 shows part of this form), which is used by physicists and therapists during planning and treatment setup. The major improvements of the revised simulation form include: 1) In the standard setup, a Silverman headrest (CIVCO Medical Solutions) placed on the pad is used for AP position, and a pediatric OR foam prone pillow off the pad is used for PA position, to ensure a straight airway in both positions. Depending on the age and size of the patient, anesthesiologists may require other support devices instead of the standard ones. The revised form clearly defines "standard" and provides a designated space to detail any deviations from the standard setup. 2) The TBI MU calculation utilizes the SSD. To accommodate the possible variety of head holders that can affect the SSD distance, the distance from the top of the head to the top of the table, including whatever head holder used, is measured both for supine $\left(\mathrm{H}_{\mathrm{ap}}\right)$ and prone $\left(\mathrm{H}_{\mathrm{pa}}\right)$ patient positions. The revised form provides a clear drawing of the setup to avoid possible confusion as to how the measurements should be taken. 3) Photos for patient setup (such as shown in Figure 1) during simulation are taken and recorded in the simulation form as a reference for dosimetry planning and treatment setup.

After the implementation of the revised simulation form, feedback from clinical staff indicates that the interdepartmental communication has greatly improved, especially in terms of patient setup. This improvement increases the work flow efficiency and reduces the stressful clinical situation since pediatric TBI is complicated and involves multiple teams. Most importantly, it decreases the risk of treating patients in a setup that differs from that used during the simulation or dosimetry planning, which could lead to treatment errors.

\subsection{New Compensator Design Method}

In the previous procedure, the compensator thickness was determined based on histor- 
(a)

\section{Standard AP Setup (check if used)}

7-cm blue pad (under both the body and the head)

$\square$ A for supine headrest

Deviations from Standard Setup (please check and specify)

$\square$

$\square$

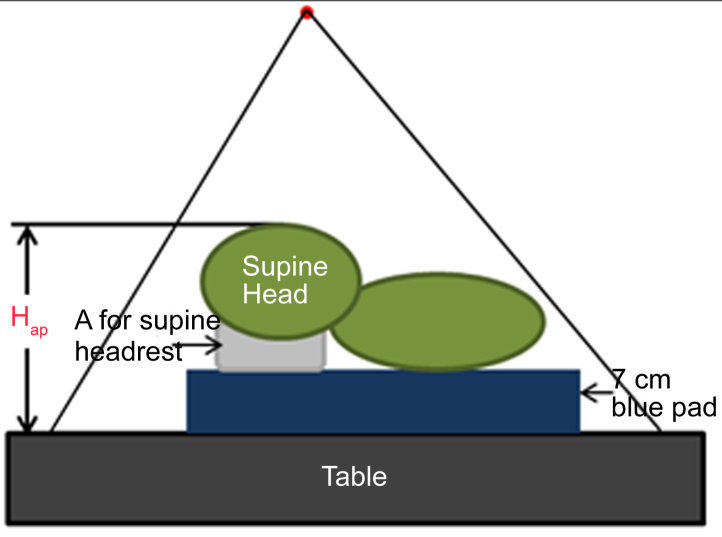

Standard AP Setup

(b)

Standard AP Setup (check if used)

$\square$ 7-cm blue pad (under the body only, not under the head)

$\square$ Pediatric OR foam prone pillow

Deviations from Standard Setup (please check and specify)

$\square$

$\square$

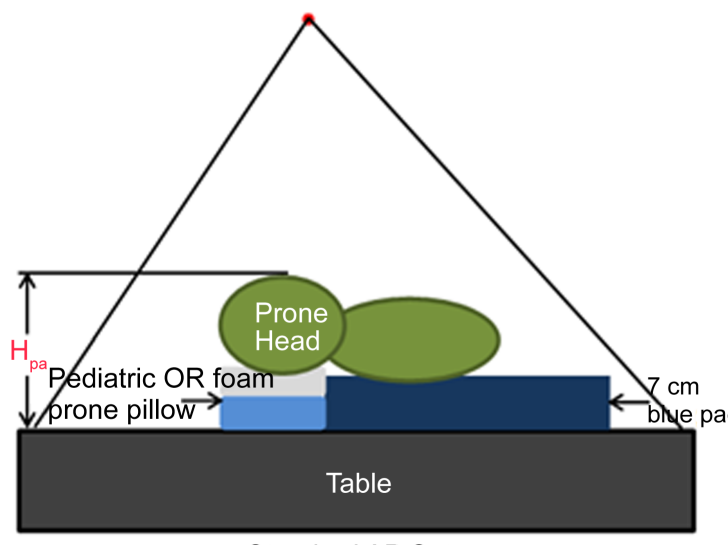

Standard AP Setup

Figure 3. The sketch above is the standard AP and PA setup instruction from the simulation form. Note the clear standard setup defined as well as areas provided to detail deviations from standard.

ical patient data, thus similar compensation was used for pediatric patients of similar size. In the new procedure, dose to the midline for each body site is first calculated using the total MUs prescribed and the PDD at the midline. The dose difference to the prescription point is then determined. The attenuation coefficient for a $1 / 4$-inch layer of acrylic compensator is $1.76 \%$, by measurement under treatment conditions. Therefore the amount of compensator is determined by calculating the dose difference for each body site and the attenuation coefficient using Equation (3), where $N$ is the number of 1/4-inch layer of acrylic compensator, $\mathrm{MU}_{\text {site }}$ is the calculated $\mathrm{MU}$ for each body site and $\mathrm{MU}_{\mathrm{p}}$ is the calculated $\mathrm{MU}$ for the prescription point.

$$
N=\frac{\frac{\mathrm{MU}_{\text {site }}}{\mathrm{MU}_{\mathrm{P}}}-1}{0.0176}
$$

To assess whether the new compensator design method is an improvement, calculations were performed on 28 previously-treated patients to determine the dose unifor- 
mity with the new versus previous method.

Data are presented as mean \pm SD. Significant differences between groups were determined using a two-tailed Student's $t$ test ( $\mathrm{p}<0.05$ was considered statistically significant). Figure 4 demonstrates that the new compensator calculation method significantly improved patient dose uniformity: $0.8 \% \pm 0.4 \%$ (new method) vs. $4.2 \% \pm 2.3 \%$ (previous method) $(\mathrm{p}<0.01)$. The data indicates that the new compensator procedure will result in fewer out-of-tolerance in vivo dosimetry readings and reduce associated re-planning efforts, which is especially critical for single-fraction treatments.

\subsection{New Treatment Setup Guidance}

In the new procedure, we also clarified the patient position with respect to the beam light field edge during treatment at extended distance. For a patient treated with the no-gap technique, formal guidelines as shown in Figure 5 were developed to set up the patient based on dosimetric profiles. The light field region corresponding to the treatment field is $\pm 52 \mathrm{~cm}$ from the central axis, but the dose falls off very quickly in the field edge due to beam penumbra. From $40 \mathrm{~cm}$ to $50 \mathrm{~cm}$ off central axis, the dose would drop from $98 \%$ to about $67 \%$ of the prescribed dose. Therefore the patient is placed in the "safe zone" region, $\pm 40 \mathrm{~cm}$ from the central axis to prevent underdose in the cranial and caudal directions. The left and right side of the patients also should be at least 12 $\mathrm{cm}$ within the visible light field edge.

\section{Discussion}

Pediatric TBI is a relatively rare and complex procedure requiring administration of anesthesia to provide adequate immobilization, thus ensuring safe treatments. Due to its infrequency, staff may be less familiar with the procedure compared to other routine treatment procedures. In addition, pediatric TBI patients come in all shapes and sizes; accommodating that wide variation in a robust process under anesthesia requires de-

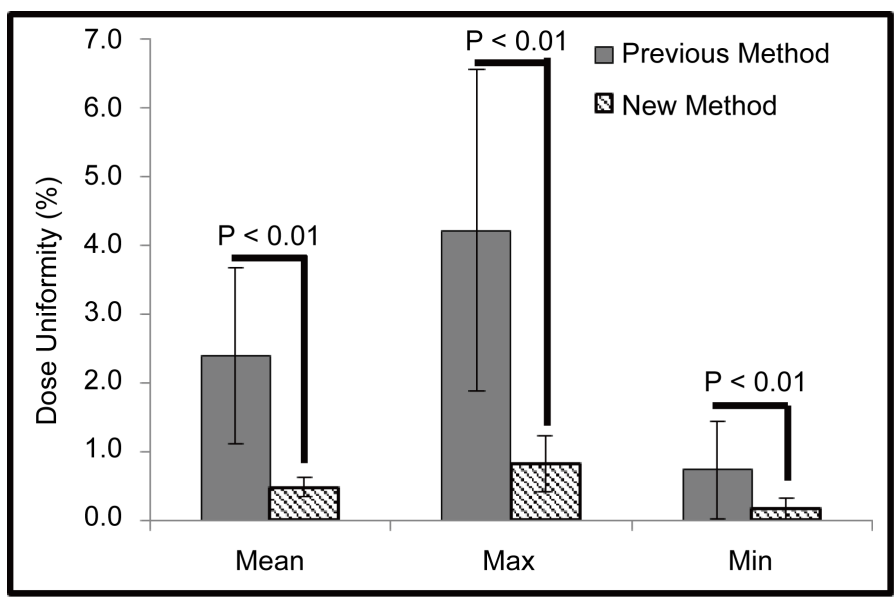

Figure 4. Comparison of the mean, maximum, and minimum dose uniformity of historical patient data $(n=28)$ using the previous and new method for compensator design. 


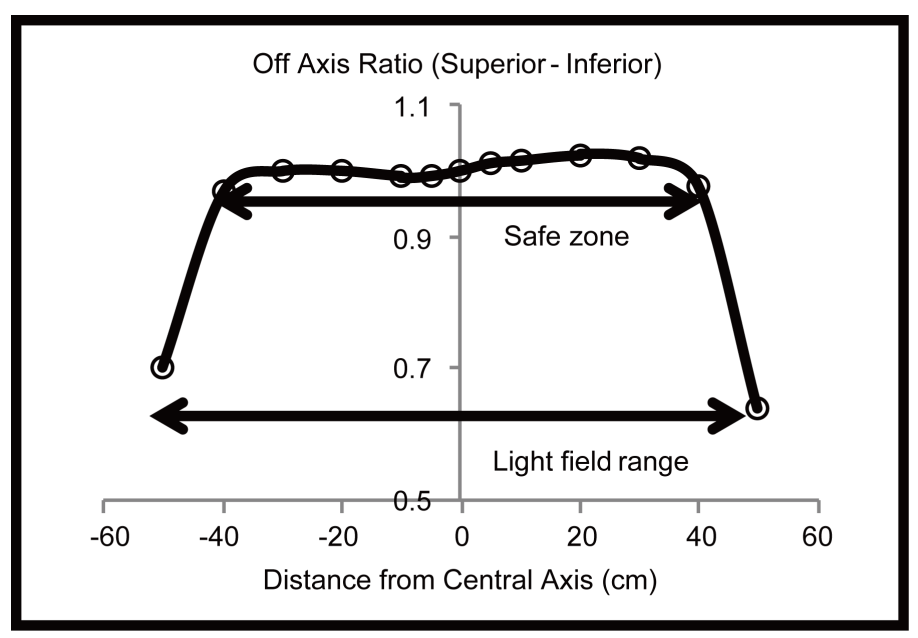

Figure 5. The plot shows the off-axis ratio measurement along patient superior-to-inferior direction.

tailed documentation and flexible dose computations to reduce the risk of errors. AAPM TG100 [8] and American Society of Radiation Oncology (ASTRO) accreditation standards strongly suggest safety reviews of the procedures in clinical practice to ensure patient safety. The technique improvements of our pediatric TBI procedure were motivated by our department's near-miss incident reporting system, implemented to improve patient safety and quality improvement.

Since pediatric patients are still in the process of growing and have a long life expectancy, TBI treatment usually has the potential to affect more organ systems for a longer period of time than in adult TBI patients. Therefore extreme care is needed for pediatric TBI cases to prevent possible mistakes, especially those mistakes that may cause large variation of overall dose distribution. The risk of relapse will increase if underdosage occurs, while the rate of morbidity will increase if overdosage of critical organs occurs [9].

Compared to the previous compensator design method, the new method more closely compensates for individual patient anatomy. Although the thickness of various body parts of a pediatric patient does not vary as much as those of an adult, it is still important to account for the individual difference. Although the TG29 report suggests $\pm 10 \%$ dose uniformity for TBI treatment [5], it is important to keep the dose variation as minimal as possible, as uncertainties add from various factors such as daily machine output, patient setup, and OSLs response. This is especially important for pediatric patients.

Pediatric TBI treatment is at extended distance with the spoiler setup. Positioning of the patient in the wooden stand relies on the light field. However, the guidelines about "flash" from the light field were not obvious. Figure 5 shows that dose drops off quickly in the edge of the light field; therefore clear instruction about patient position with respect to beam light field edge is implemented in the new procedure to avoid the risk that part of patient may be placed in the penumbra region. 
Table 1. Differences between adult and pediatric TBI treatment in our clinic.

\begin{tabular}{ccc}
\hline & Adult & Pediatric \\
\hline Energy & $18 \mathrm{MV}$ & $6 \mathrm{MV}$ \\
Source-Skin-Distance & $475 \mathrm{~cm}$ & $190 \mathrm{~cm}$ \\
Patient position & Standing AP/PA & Prone/Supine AP/PA \\
Compensator & Decubitus AP/PA & Acrylic on top of floor stand \\
Blocking (lung) & Lead in tray of machine & Cerrobend placed on acrylic top \\
Anesthesia & Cerrobend hung on spoiler tray & Yes \\
\hline
\end{tabular}

This AP/PA method is straightforward and easily adopted by other clinics and achieves good dose uniformity for pediatric TBI patients who require anesthesia, usually ranging in age from infancy to 7 years. Those pediatric patients who are cooperative are treated in the standing position with AP/PA beams using the typical adult stand [10]. The differences between adult and pediatric TBI treatment in our clinic are listed in Table 1. The lateral treatment technique in which patients are treated with opposed lateral beams provides great positional stability; however, $10 \%$ dose uniformity can be achieved only for patients with a lateral separation thickness of less than $45 \mathrm{~cm}$ [11]. In general, better dose uniformity can be achieved with the AP/PA technique [6]. Although the recently developed helical tomotherapy treatment method for TBI can provide adequate target coverage with individual sparing of organs at risk such as the lungs, there are potential drawbacks such as more complicated planning, prolonged overall treatment time, high instantaneous dose rate for tissue elements, and access to the technology [12] [13].

\section{Conclusion}

A near-miss incident reporting system was used to improve the safety and quality of pediatric TBI procedures under anesthesia. For an institution averaging one pediatric TBI case per one and half months, standardization of and other changes to the procedure simplify the planning process, improve patient safety, and improve dose uniformity. Since there are few reports about the procedures and techniques involved in pediatric TBI requiring anesthesia, this experience may be especially beneficial for other centers with lower volumes of pediatric TBI patients.

\section{References}

[1] Pasquini, M.C. and Wang, Z. (2009) Current Use and Outcome of Hematopoietic Stem Cell Transplantation: Part I-Cibmtr Summary Slides. CIBMTR Newsletter [Serial Online], 15, 7-11.

[2] Bunin, N., Aplenc, R., Kamani, N., Shaw, K., Cnaan, A. and Simms, S. (2003) Randomized Trial of Busulfan vs Total Body Irradiation Containing Conditioning Regimens for Children with Acute Lymphoblastic Leukemia: A Pediatric Blood and Marrow Transplant Consortium Study. Bone Marrow Transplantation, 32, 543-548.

http://dx.doi.org/10.1038/sj.bmt.1704198 
[3] Davies, S.M., Ramsay, N.K., Klein, J.P., Weisdorf, D.J., Bolwell, B., Cahn, J.Y., Camitta, B,M., Gale, R.P., Giralt, S., Heilmann, C., Henslee-Downey, P.J., Herzig, R.H., Hutchinson, R., Keating, A., Lazarus, H.M., Milone, G.A., Neudorf, S., Perez, W.S., Powles, R.L., Prentice, H.G., Schiller, G., Socié, G., Vowels, M., Wiley, J., Yeager, A. and Horowitz, M.M. (2000) Comparison of Preparative Regimens in Transplants for Children with Acute Lymphoblastic Leukemia. Journal of Clinical Oncology, 18, 340-347.

[4] Aschan, J. (2007) Risk Assessment in Haematopoietic Stem Cell Transplantation: Conditioning. Best Practice \& Research Clinical Haematology, 20, 295-310. http://dx.doi.org/10.1016/j.beha.2006.09.004

[5] Dyk, L.V., Galvin, J.M., Glasgow, G.P. and Podgorsak, E.B. (1986) The Physical Aspects of Total and Half Body Photon Irradiation. AAPM Report No. 17 (Task Group 29), American Institute of Physics, College Park.

[6] Halperin, E.C., Brady, L.W., Perez, C.A. and Wazer, D.E. (2013) Perez and Brady's Principles and Practice of Radiation Oncology. 6th Edition, Lippincott Williams \& Wilkins, Philadephia.

[7] Yukihara, E.G., Mardirossian, G., Mirzasadeghi, M., Guduru, S. and Ahmad, S. (2008) Evaluation of $\mathrm{Al}_{2} \mathrm{O}_{3}$ : C optically Stimulated Luminescence (OSL) Dosimeters for Passive Dosimetry of High-Energy Photon and Electron Beams in Radiotherapy. Medical Physics, 35, 260-269. http://dx.doi.org/10.1118/1.2816106

[8] Huq, M.S., Fraass, B.A., Dunscombe, P.B., Gibbons Jr., J.P., Ibbott, G.S., Mundt, A.J., Mutic, S., Palta, J.R., Rath, F., Thomadsen, B.R., Williamson, J.F. and Yorke, E.D. (2016) The Report of Task Group 100 of the AAPM: Application of Risk Analysis Methods to Radiation Therapy Quality Management. Medical Physics, 43, 4209.

http://dx.doi.org/10.1118/1.4947547

[9] Vollans, S.E., Perrin, B., Wilkinson, J.M., Gattamaneni, H.R. and Deakin, D.P. (2000) Investigation of Dose Homogeneity in Paediatric Anthropomorphic Phantoms for a Simple Total Body Irradiation Technique. The British Journal of Radiology, 73, 317-321. http://dx.doi.org/10.1259/bjr.73.867.10817050

[10] Wills, C., Cherian, S., Jacob Yousef, J., Wang, K. and Mackley, H.B. (2016) Total Body Irradiation: A Practical Review. Applied Radiation Oncology, 5, 11-17.

[11] Mesa, F., Eng, T.Y., Esquivel, C., Fuller, C.D., Papanikolaou, N. and Sosa, M. (2011) Implementation of a Lateral Total Body Irradiation Technique with 6 MV Photons: The University of Texas Health Science Center in San Antonio Experience. Journal of Radiotherapy in Practice, 10, 45-54. http://dx.doi.org/10.1017/S1460396910000221

[12] Hui, S.K., Kapatoes, J., Fowler, J., Henderson, D., Olivera, G., Manon, R.R., Gerbi, B., Mackie, T.R. and Welsh, J.S. (2005) Feasibility Study of Helical Tomotherapy for Total Body or Total Marrow Irradiation. Medical Physics, 32, 3214-3224. http://dx.doi.org/10.1118/1.2044428

[13] Gruen, A., Ebell, W., Wlodarczyk, W., Neumann, O., Kuehl, J.S., Stromberger, C., Budach, V. and Marnitz, S. (2013) Total Body Irradiation (TBI) Using Helical Tomotherapy in Children and Young Adults Undergoing Stem Cell Transplantation. Radiation Oncology, 8, 92. http://dx.doi.org/10.1186/1748-717X-8-92 